\title{
Effects of Prolonged Waking-Auditory Stimulation on Electroencephalogram Synchronization and Cortical Coherence during Subsequent Slow-Wave Sleep
}

\author{
Jose L. Cantero, Mercedes Atienza, Rosa M. Salas, and Elena Dominguez-Marin \\ Laboratory of Sleep and Cognition, 41005 Seville, Spain
}

Evidence suggests that sleep homeostasis is not only dependent on duration of previous wakefulness but also on experience- and/or use-dependent processes. Such homeostatic mechanisms are reflected by selective increases in the duration of a sleep stage, modifications to electrophysiological-metabolic brain patterns in specific sleep states, and/or reactivation to neuronal ensembles in subsequent sleep periods. Use-dependent sleep changes, apparently different from those changes caused by memory consolidation processes, are thought to reflect neuronal restoration processes after the sustained exposure to stimulation during the preceding wakefulness. In the present study, we investigated changes in the brain electrical activity pattern during human sleep after $6 \mathrm{hr}$ of continuous auditory stimulation during previous wakefulness. Poststimulation nights showed a widespread increase of spectral power within the $\alpha(8-12 \mathrm{~Hz})$ and sleep spindle $(12-15 \mathrm{~Hz})$ frequency range during slow-wave sleep (SWS) compared with the baseline night. This effect was mainly attributable to an enhanced EEG amplitude rather than an increase of oscillations, except for temporal (within $\alpha$ and sleep spindles) and parietal regions (within sleep spindles) in which both parameters contributed equally to the increase of spectral energy. Power increments were accompanied by a strengthening of the coherence between fronto-temporal cortical regions within a broad frequency range during SWS but to the detriment of the coherence between temporal and parieto-occipital areas, suggesting underlying compensatory mechanisms between temporal and other cortical regions. In both cases, coherence was built up progressively across the night, although no changes were observed within each SWS period. No electrophysiological changes were found in rapid eye movement sleep.

These results point to SWS as a critical brain period for correcting the cortical synaptic imbalance produced by the predominant use of specific neuronal populations during the preceding wakefulness, as well as for synaptic reorganization after prolonged exposure to a novel sensory experience.

Key words: slow-wave sleep; sleep spindles; $\alpha$ activity; spectral analysis; coherence; sleep homeostasis; use-dependent function of sleep
Cerebral activity during mammalian sleep has been proposed to arise from activation of wakefulness-dependent homeostatic mechanisms, as well as from circadian and ultradian processes (Tobler et al., 1992). Early studies demonstrated that an extended period of wakefulness produces an augmentation of spectral density in the low-frequency bands during recovery non-rapid eye movement (NREM) sleep (Borbély et al., 1981). This finding suggests that brain mechanisms responsible for slow-wave activity generation are extremely sensitive to sleep pressure and may be the most likely cause for cerebral homeostasis after sleep deprivation (Borbély, 1982).

Growing evidence suggests that electrophysiological changes during slow-wave sleep (SWS) are also homeostatic responses to intense activation of specific neuronal groups during wakef ulness as a result of novel behavioral events. For instance, García-García et al. (1998) found an increased spectral power in $\delta(0.25-4 \mathrm{~Hz})$, $\theta(4.25-8 \mathrm{~Hz})$, and the $12-16 \mathrm{~Hz}$ frequency range in rats during $4 \mathrm{hr}$ of sleep after a forced wakefulness period induced by gentle handling, excess of food intake, or a stressful immobilization. In humans, Kattler et al. (1994) showed that unilateral stimulation of the left somatosensory cortex during $6 \mathrm{hr}$ of wakefulness resulted

Received Dec. 28, 2001; revised Feb. 25, 2002; accepted March 11, 2002.

Correspondence should be addressed to Dr. Jose L. Cantero, Laboratory of Sleep and Cognition, Avenida de Andalucia 16, 1D-Izquierda, 41005 Seville, Spain. E-mail: jose_cantero@hms.harvard.edu.

Copyright $\odot 2002$ Society for Neuroscience $0270-6474 / 02 / 224702-07 \$ 15.00 / 0$ in a significant shift of the interhemispheric asymmetry over central regions for the $0.75-4.5 \mathrm{~Hz}$ frequency range during the first hour of NREM sleep relative to baseline. Similar results were found in rats after cutting the whiskers on one side to reduce the sensory inputs in the contralateral cortex (Vyazovskiy et al., 2000). In both studies, increments of spectral power within approximately the same frequency range were shown during sleep over those cortical regions that had been more intensely stimulated during the preceding wakefulness, suggesting selective homeostatic changes in the neuronal function attributable to the sustained stimulation during the previous waking period.

The neural substrates underlying the use-dependent function of sleep remain elusive, however, especially because of the intrinsic difficulties in differentiating between use- and learningdependent sleep modulations (Maquet, 2001). To begin addressing this question, it would be necessary to explore whether overstimulation of cortical regions different from somatosensory areas affects sleep electrophysiology. This can be tested by presenting the subjects with stimulation of another sensory modality. Previous studies have shown changes in sleep architecture after both prolonged acoustic stimulation (Cantero et al., 2002) and auditory deprivation (Pedemonte et al., 1997), suggesting that the auditory modality is a reliable experimental tool to test the use-dependent hypothesis of sleep function.

According to this hypothesis, sustained activation of auditory cortical regions during wakefulness should influence the brain 
activity patterns during subsequent sleep period, as reflected by changes in scalp EEG activity compared with the baseline night. Such changes at the level of the cortex during sleep are thought to maintain the synaptic efficacy of those neuronal populations that were understimulated during waking (Krueger et al., 1995) to stabilize experience-induced new synapses (Kavanau, 1997) or to simply reflect the restoration of an optimal neuronal function after the sustained waking neuronal activity (Maquet, 2001). The absence of such electrophysiological changes after the prolonged waking-auditory stimulation would suggest that use-dependent synaptic modifications are only restricted to certain cortical regions after the persistent stimulation of the somatosensory system. On the contrary, the use-dependent sleep-related changes could be a generalized property of brain function in response to dramatic modifications of cerebral metabolism during previous wakefulness. To test this hypothesis, we studied scalp EEG modulations during human sleep after continuous auditory stimulation administered during the preceding 6 wake-time hours.

\section{MATERIALS AND METHODS}

Subjects. Eight volunteer subjects (five males and three females; aged 20-29 years old; mean \pm SD age, $24.8 \pm 2.42$ years), recruited among university students, participated in the present study. All of them were right-handed with normal hearing. Subjects were screened for health status with a structured medical interview. Medical illness, psychiatricpsychological disturbance, substance abuse, and/or neurological disorders were criteria for exclusion. Subjects were also screened for any history of sleep disorders using sleep questionnaires. They were instructed to maintain a normal sleep-wake schedule and refrain from alcohol, caffeine, medication, and drug consumption during $48 \mathrm{hr}$ before each experimental session. Sleep logs indicated no variations in sleep parameters in those nights preceding baseline and experimental sessions. No naps were reported by any of the subjects during the entire period of the study. Written informed consent was obtained from all participants after detailed explanation of the experimental protocol.

Experimental protocol. Subjects slept four nights in the sleep laboratory. Nights 1 and 2 (consecutive) were considered as adaptation and baseline nights, respectively. Both adaptation and baseline nights were also used to objectively determine sleep abnormalities (excessive sleep fragmentation, prolonged sleep latency, REM sleep onset, etc. . ), which could be indicative of sleep disorders. Remaining nights ( 3 and 4 ) were preceded by an auditory stimulation session (right and left, respectively). Stimulation was presented for $6 \mathrm{hr}$ during the preceding wakefulness (from 4:00 P.M. to 10:00 P.M.). The interval between experimental sessions was 1 week to avoid carryover effects of the auditory stimulation on sleep. Because the auditory system is bilaterally organized, unilateral inputs should affect homologous subcortical and cortical auditory structures in a similar way (Webster et al., 1992). Thus, one poststimulation night was used as control for the other because identical effects on EEG sleep are expected after either right or left auditory stimulation.

Auditory stimulation. Acoustic stimuli were presented while subjects sat comfortably in a sound-attenuated room, reading a book of their own preference. Subjects were instructed to ignore auditory stimulation. When stimuli were administered by one channel, the contralateral ear was isolated from any external stimulation with wax earplugs and secured with surgical tape. Four different auditory patterns of $250 \mathrm{msec}$ each were presented unilaterally via insert earphones (Etymotic Research, Elk Grove Village, IL) at $70 \mathrm{~dB}$ sound pressure level, with an interstimulus interval of $300 \mathrm{msec}$. All patterns resulted from the spectral summation of five harmonic tones: first $(350,700,1400,2800$, and $5600 \mathrm{~Hz})$, second $(400,800,1600,3200$, and $6400 \mathrm{~Hz})$, third $(450,900,1800,3600$, and 7200 $\mathrm{Hz})$, and fourth $(500,1000,2000,4000$, and $8000 \mathrm{~Hz})$. Thus, activation of different neuronal groups in the primary auditory cortex was ensured based on the tonotopic organization of this brain structure in the processing of complex sounds (Rauschecker et al., 1995). Each pattern was presented alone in different blocks for $13 \mathrm{~min}$, the interblock interval being $2 \mathrm{~min}$. The order in which the four blocks were presented was counterbalanced within the session but remained constant from one experimental session to the next.

Electroencephalographic data acquisition. Polysomnographic recordings were performed in an acoustically shielded room from 12:00 A.M. (lights off) to 8:00 A.M. (lights on). The EEG was continuously recorded from 23 scalp locations (Fp1, Fp2, F3, F4, F7, F8, Fz, C3, C4, Cz, P3, P4, Pz, T3a, T3, T5a, T5, T4a, T4, T6a, T6, O1, and O2) and referenced to a linked-mastoid derivation. Vertical ocular movements were recorded with a pair of electrodes placed above and below the left eye and horizontal electrooculography with another pair $1 \mathrm{~cm}$ apart from the outer canthi of each eye, respectively. Electromyography was also bipolarly recorded from chin muscles (3 cm apart). Electrophysiological measurements were recorded using silver-silver chloride disk electrodes filled with electrode cream and attached with either surgical tape (face placements) or collodion (scalp placements). All electrophysiological variables were amplified, filtered (bandpass, $0.5-50 \mathrm{~Hz},-3 \mathrm{~dB}$ points of a $24 \mathrm{~dB}$ per octave roll-off curve), digitized ( $200 \mathrm{~Hz}, 12$-bit resolution), and stored in digital format for off-line analysis. Electrode-skin impedance was kept below $5000 \Omega$. The same recording protocol was used in the four nights required per subject (adaptation, baseline, right, and left stimulation). Each recording was randomly assigned to two technicians, who were not informed of the purpose of the investigation, for sleep scoring. The two scorers were from the same laboratory and had received the same training, although the length of their experience differed. Disputed epochs were blind scored to the experimental aims, one-by-one by another different technician strictly according to standardized rules for human sleep staging (Rechtschaffen and Kales, 1968).

Calculation of power and coherence spectra. Artifact-free EEG epochs (5.12 sec each) were manually selected during SWS (stages 3 and 4) and REM sleep in each night (baseline, right, and left stimulation). EEG segments containing clipped channels, oculomotor activity (slow or rapid eye movements), arousals, and/or transient increases in the EMG channel were excluded from additional analysis. Averaged power density spectra were estimated for SWS and REM sleep in each night for all EEG derivations using a fast Fourier transform algorithm. The frequency resolution was set at $0.2 \mathrm{~Hz}$, and only frequencies up to $40 \mathrm{~Hz}$ were analyzed. Data reduction was achieved by averaging values over five adjacent $0.2 \mathrm{~Hz}$ frequency bins.

It is well known that power measurements provided by the spectral analysis can be the result of an increase in either the wave incidence or the wave amplitude. To dissociate between both sources, period-amplitude analysis technique was applied to the poststimulation nights in those EEG derivations and frequency bands in which spectral power increments were statistically different from the baseline night. Period-amplitude technique is composed by two different analysis procedures. First, the EEG signal is examined to detect whether a zero-crossing event has occurred. Zero-crossing events are defined as changes in the polarity of the EEG signal crossing $0 \mathrm{~V}$ threshold. Each time a zero-crossing event occurs in one EEG epoch, the duration since the last zero-cross event is determined, and the frequency is then identified. Second, the area under the curve, or integrated amplitude, is calculated as a measurement of amplitude within a frequency range. These parameters, zero crossing and integrated amplitude, provide information about the number of oscillations and the wave amplitude, respectively, in a specific EEG derivation for any frequency band of interest. Occasionally, period-amplitude algorithms do not detect fast EEG frequencies superimposed on a background of slow-wave activity. To solve this problem, applying a bandpass filter to the signal before period-amplitude analysis has been suggested (Ktonas, 1987). The period-amplitude algorithm used here was designed with MATLAB (MathWorks, Natick, MA) and has been described in detail previously (Hoffmann et al., 1979).

Cortical coherences from human scalp recordings provide large-scale measures of functional interrelations between pairs of neocortical regions, yielding information about network formation and brain binding (Nunez et al., 1997). The coherence function is defined as the squared normalized cross-power spectrum and measures the phase consistency between pairs of signals within each frequency band (Jenkins and Watts, 1968). Coherence analysis was applied to equally spaced bipolar derivations to avoid the undesirable effects of a common reference electrode (Essl and Rappelsberger, 1998) and to increase the sensitivity in detecting cortico-cortical functional coupling underlying both local and global EEG dynamics (Nunez et al., 2001). Choosing bandwidths requires making a compromise between data stationarity, frequency resolution, and statistical significance of coherence estimates (Nunez et al., 1999). Because of these factors, $1 \mathrm{~Hz}$ bins were initially used for the computation of coherence spectra $(1-40 \mathrm{~Hz})$ in the following intrahemispheric bipolar pairs: F3C3-P3O1, T3aT3-T5aT5, F3C3-T3aT3, F3C3-T5aT5, P3O1-T3aT3, P3O1-T5aT5, F4C4-P4O2, T4aT4-T6aT6, F4C4-T4aT4, F4C4-T6aT6, P4O2-T4aT4, and P4O2-T6aT6. Interhemispheric coher- 
ences were also computed for F3C3-F4C4, P3O1-P4O2, T3aT3-T4aT4, and T5aT5-T6аT6.

The time course of the EEG coherence across the night was also investigated within and between sleep cycles during the poststimulation nights in those pairs of electrodes and frequency ranges that showed statistical differences compared with the baseline night. To study the temporal dynamic of coherence within a sleep cycle, sets of 20 artifactfree EEG epochs (5.12 sec each) were selected at the beginning, middle, and end of each sleep cycle. Coherence analysis was independently applied to each set of EEG epochs, providing the possibility of comparing coherence values in three critical points of a same sleep cycle. In a different analysis, one coherence measurement was computed per sleep cycle to examine the temporal evolution of coherence between the different sleep cycles across the night.

Statistical analyses. Possible effects of unilateral auditory stimulation on lateralization of spectral energy during different sleep states were evaluated by means of an interhemispheric asymmetry index (IAI) (Gasser et al., 1988). This index consists of dividing spectral power density over the left hemisphere by the sum of spectral power density over the left and right hemispheres. The IAI was calculated separately for each frequency component $(1-40 \mathrm{~Hz})$ and sleep state (SWS and REM sleep) in the following cortical areas: frontal (F3 vs F4), central (C3 vs C4), temporal-1 (T3a vs T4a), temporal-2 (T3 vs T4), temporal-3 (T5a vs T6a), temporal-4 (T5 vs T6), parietal (P3 vs P4), and occipital (O1 vs O2). One-way ANOVAs for repeated measurements (rANOVAs) were performed on IAI values with factor "night" (baseline, left, and right) for each frequency bin and sleep state. To simplify the data set, homologous EEG derivations were collapsed if no significant changes of the interhemispheric asymmetry were found.

For statistical purposes, spectral power was logarithmically transformed. The effect of prolonged auditory stimulation on log EEG spectral power obtained during different sleep states was tested with one-way rANOVAs (night factor). Independent rANOVAs were performed for each combination of scalp region (frontal, central, temporal, parietal, and occipital) and sleep state (SWS and REM sleep) in each frequency component $(1-40 \mathrm{~Hz})$. Period-amplitude technique was applied to those EEG derivations and frequency ranges showing statistical differences between poststimulation and baseline nights as revealed by the spectral analysis technique. In these cases, independent one-way rANOVAs (night factor) were performed for each analysis method composing this technique (zero crossing and integrated amplitude), frequency band, and cortical region.

Fisher $z$-transformation $\left(z=\tanh ^{-1}\right)$ was applied to coherence data for a best fit with a normalized distribution, making the use of parametric statistics possible. Independent one-way rANOVAs (night factor) were performed on each coherence pair and sleep state (SWS and REM sleep) for each frequency component $(1-40 \mathrm{~Hz})$. The temporal course of EEG coherence was also explored within and between sleep cycles, considering those pairs of electrodes and frequency bands showing significant differences between experimental and baseline nights in the previous coherence analysis. To examine whether the temporal evolution of these coherences builds up progressively within a sleep cycle, independent two-way ("time course" $\times$ "sleep cycle" factors) rANOVAs were performed for each significant coherence and frequency range. One-way ("sleep cycle" factor) rANOVAs were applied to explore the temporal course of the coherence between the different sleep cycles across the night.

Conservative Greenhouse-Geisser correction of freedom degrees was applied, and the null hypothesis was rejected with probability values below 0.05 in all rANOVAs. Post hoc contrasts for each possible combination of two nights were performed by applying paired $t$ tests $(p<0.05)$ where necessary.

\section{RESULTS}

Although statistical analysis were always performed from 1-40 $\mathrm{Hz}$, no significant differences between baseline and poststimulation nights were found with any quantitative EEG technique above $20 \mathrm{~Hz}$; hence, figures only show data within the $1-20 \mathrm{~Hz}$ range of the spectrum.

\section{Interhemispheric asymmetry}

Statistical analysis revealed no significant effects in the entire spectrum (1-40 Hz) on regional scalp EEG asymmetries both in
SWS and REM sleep when comparing baseline and nights preceded by $6 \mathrm{hr}$ of auditory stimulation. Because prolonged unilateral presentation of auditory stimuli during wakefulness did not generate an asymmetric EEG pattern in the subsequent sleep, homologous electrodes were collapsed for additional analyses.

\section{EEG power spectra}

Figure 1 depicts averaged all-night EEG power spectra obtained from baseline and experimental nights during both SWS and REM sleep from different scalp regions. Black bars below power spectra denote post hoc comparisons between baseline and each experimental night, revealing a significant higher amount of spectral power in those nights preceded by a session of auditory stimulation. Specifically, a significant increase of absolute power in the $\alpha$ and fast sleep spindle range was observed in both experimental nights in $66.7 \%$ of the obtained main effects, these results being replicated in $88.1 \%$ of the total population. Of the remaining main effects, $23.3 \%$ were attributable to spectral power increases only after right stimulation (replicated in $90 \%$ of the total population) and $10 \%$ only after left stimulation (replicated in $100 \%$ of the population). No significant differences in spectral power were found in REM sleep when baseline and experimental nights were compared.

Figure 2 shows the mean percentage increases in the spectral power, wave incidence, and wave amplitude during the nights after acoustic overstimulation compared with baseline night in those cortical regions (central, parietal, occipital, and temporal) in which overall rANOVAs revealed main effects. Significant differences were restricted to SWS, extended over almost the whole scalp, and mainly clustered around those frequency components corresponding to $\alpha$ activity and sleep spindles.

The enhanced spectral power found during SWS was mainly attributable to a significant increase of the wave amplitude. Oneway rANOVAs comparing the integrated amplitude values among nights yielded significant main effects within the $\alpha$ and sleep spindle frequency range widespread over the scalp (central, temporal, parietal, and occipital). Post hoc $t$ tests confirmed that wave amplitudes were always higher during nights after auditory stimulation compared with baseline ( $p$ values ranged from 0.00002 to 0.05 ). The enhanced spectral power within the $\alpha$ and sleep spindle band was further observed to be partially attributable to a significant increase in the wave incidence over temporal regions for both frequency bands $\left(\alpha: F_{(2,14)}=4.57, p<0.035, \epsilon=\right.$ 0.901 ; right stimulation vs baseline: $t=-2.81, p<0.02$; left stimulation vs baseline: $t=-2.64, p<0.03$; spindles: $F_{(2,14)}=$ 3.12, $p<0.05, \epsilon=0.908$; right stimulation vs baseline: $t=-2.66$, $p<0.03$; left stimulation vs baseline: $t=-1.94, p<0.05)$ and parietal areas only for sleep spindles $\left(F_{(2,14)}=4.42, p<0.05, \epsilon=\right.$ 0.574; right stimulation vs baseline: $t=-4.30, p<0.004$; left stimulation vs baseline: $t=-2.62, p<0.03)$. Therefore, neuronal activity within the temporal cortex showed not only a higher number of oscillations but also a higher amplitude both in $\alpha$ and spindle range in the nights after the sustained acoustic stimulation compared with the baseline night. An analogous result was found over parietal areas, although restricted to the sleep spindles, suggesting a well defined influence of thalamocortical networks on parietal cortex.

\section{EEG coherence}

Averaged coherence values for those coherence pairs in which the overall rANOVAs revealed main effects among baseline and experimental nights across the entire spectrum are displayed in 

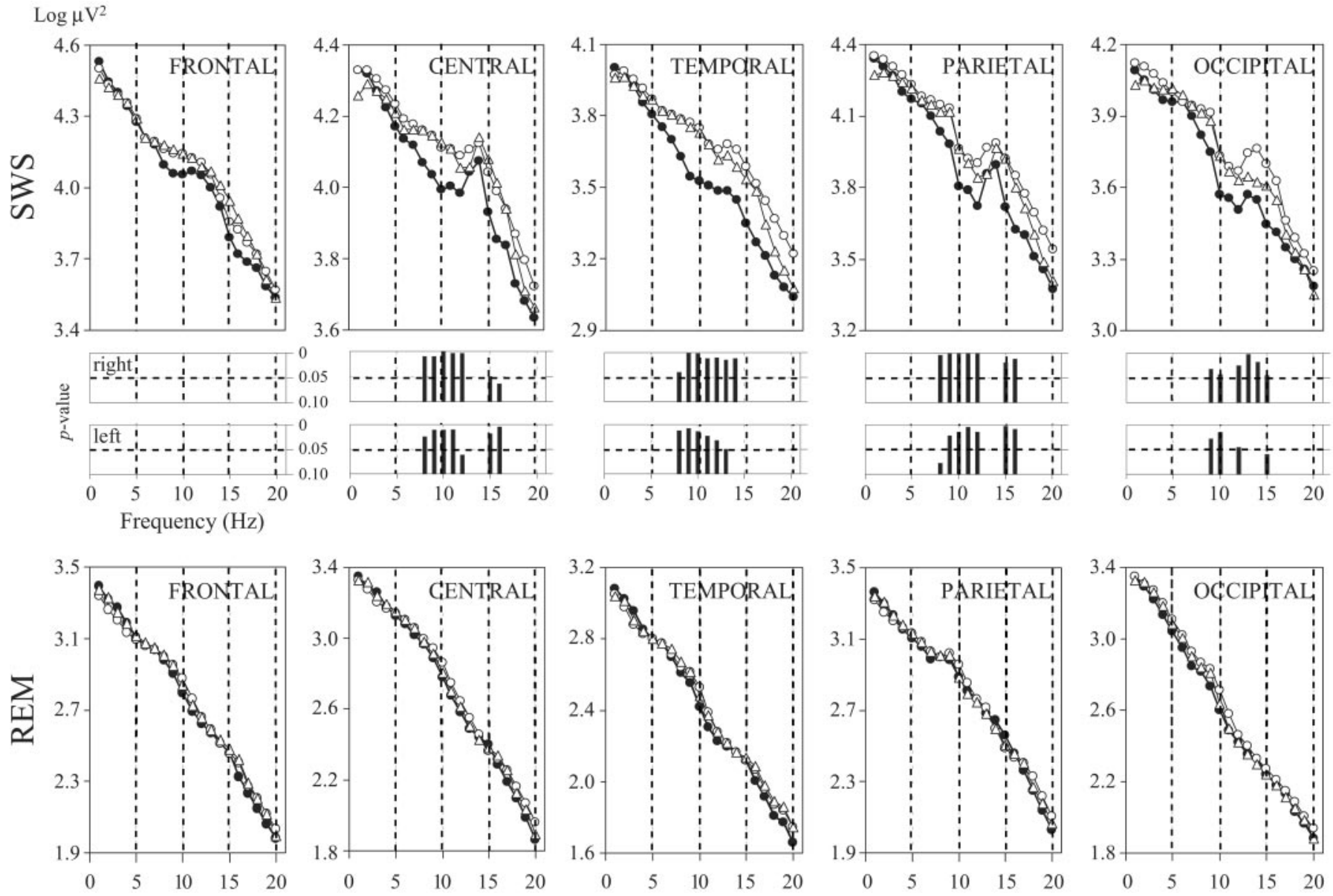

$\rightarrow$ Baseline $\multimap-$ Right $\rightarrow-$ Left

Figure 1. Mean absolute power spectra calculated for SWS and REM. Electrodes over the same brain region were collapsed (frontal: F3, F4, and Fz; central: C3, C4, and Cz; temporal: T3a, T3, T5a, T5, T4a, T4, T6a, and T6; parietal: P3, P4, and Pz; occipital: O1 and O2). Right and left labels indicate the effects of the stimulation side on spectral power during sleep. Black bars below each SWS spectrum denote significance levels (post hoc $t$ tests) between experimental (right or left) and baseline nights only when overall rANOVA showed a main effect of the night factor. Auditory stimulation in wakefulness was followed by a power increase during SWS circumscribed to the $\alpha$ and spindle ranges over almost the whole scalp, whereas no differences among nights were found during REM sleep.

Figure 3, where bars denote the post hoc significance levels when experimental and baseline nights were compared. Figure 4 shows the percentage of coherence change in different classic frequency bands for the same coherence pairs after comparing experimental and baseline night.

Prolonged auditory stimulation delivered on the right channel during the first stimulation session selectively affected the cortical coherence within the left hemisphere during the following SWS periods, specifically between left parieto-occipital (P3O1) and left posterior temporal areas (T5aT5) and between left fronto-central regions (F3C3) and left posterior temporal areas (T5aT5) when compared with the baseline night. Effects extended over a broad frequency range for each coherence pair mentioned above. However, the direction of the electrophysiological changes was different in each case. Right auditory stimulation significantly weakened cortical coherence between left posterior cortex (P3O1) and left posterior temporal regions (T5aT5) in the $\delta, \theta, \alpha$, spindles, and $\beta$ frequency bands during SWS compared with baseline (Fig. 4 ), whereas the coherence between left fronto-central cortex (F3C3) and left posterior temporal regions (T5aT5) were strengthened during SWS within the $\delta, \alpha$, spindles and $\beta$ fre- quency ranges (Fig. 4). Specifically, a significant increase in cortical coherence was observed in both experimental nights in only $8 \%$ of the obtained main effects, this effect being replicated in all subjects. Of the remaining main effects, $92 \%$ were attributable to changes in EEG coherence only after right stimulation (replicated in $91.3 \%$ of the total population). No effects were found only after left stimulation for any subject.

The effect of auditory stimulation altered the time course of changes in cortical coherence during SWS periods depending on the involved cortical areas. Thus, whereas the long-range coherence (F3O1-T5aT5) remained constant across the night, the short-distance coherence (P3O1-T5aT5) showed significant differences among sleep cycles $(p<0.003$ in all frequency bands). Post hoc tests showed that the decrease of the short-range coherence was built progressively across sleep cycles during the night after stimulation. This effect was most prominent in the two last sleep cycles compared with the baseline night. No significant changes in the building up of cortical coherence were found when effects of prolonged acoustic stimulation were studied within each sleep cycle for each coherence pair.

Cortical coherence during REM sleep was unaffected by the 
Alpha $(8-12 \mathrm{~Hz})$
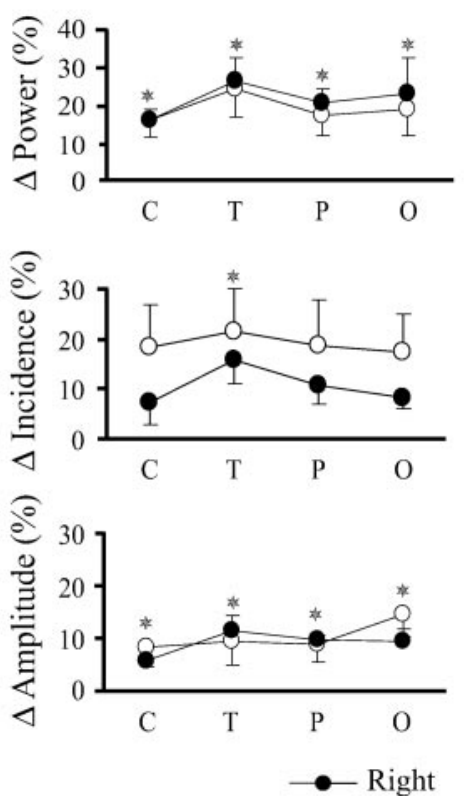

- Right
Spindles $(12-15 \mathrm{~Hz})$
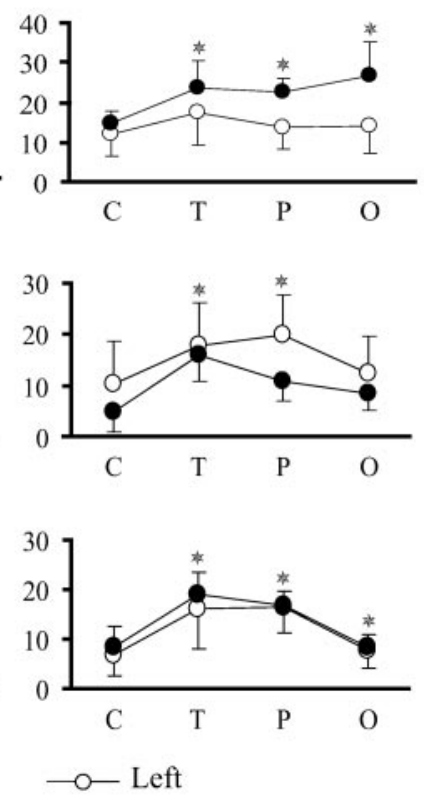

Figure 2. Mean percentage increases in the EEG spectral power, wave incidence, and wave amplitude within the $\alpha$ and spindle range during the SWS periods in nights after acoustic overstimulation compared with baseline night in only those cortical regions [central $(C)$, parietal $(P)$, occipital $(O)$, and temporal $(T)]$ in which overall rANOVAs revealed main effects. Asterisks indicate significant increase $(p<0.05)$ in both nights after auditory stimulation compared with baseline night. Note that significant increments of spectral power within $\alpha$ and sleep spindle band were mainly caused by an increase in wave amplitude, although enhanced spectral power was further observed to be partially attributable to a significant increase in the wave incidence over temporal regions for both frequency bands and parietal areas only for sleep spindles.

sustained auditory stimulation delivered during the previous wakefulness.

\section{DISCUSSION}

The present study revealed cortical electrophysiological changes restricted to SWS in those nights preceded by $6 \mathrm{hr}$ of acoustic stimulation. These changes are summarized in (1) a widespread increase of spectral power within the range of $\alpha$ activity (8-12 $\mathrm{Hz}$ ) and sleep spindles (12-15 Hz) and (2) complex modulations in the cortical coherence between the auditory cortex and other cortical regions within a broad frequency range.

\section{Sensory experience-dependent plasticity as revealed by enhanced EEG spectral power}

The increase of spectral energy both in the $\alpha$ and sleep spindle frequency range was extended over the whole scalp without an apparent lateralization effect after either right or left stimulation. Similar findings have been obtained after $30 \mathrm{~min}$ of unilateral exposure to pulsed high-frequency electromagnetic fields in the range of cellular telephones (Huber et al., 2000), resulting in increased EEG spectral power in the 9.75-11.25 and 12.25-13.25 $\mathrm{Hz}$ ranges during NREM sleep. However, the affected neural mechanisms during NREM sleep by previous stimulation seem to be dependent on the sensory modality, because prolonged unilateral somatosensory stimulation caused a shift of EEG energy in the $\delta$ frequency range toward the somatosensory region contralateral to the side of stimulation (Kattler et al., 1994). Also in agreement with previous studies, we found no electrophysiological modulations during REM sleep after auditory stimulation.

These previous findings, combined with the data from this study, suggest that homeostatic responses during sleep can be determined not only by the duration of the previous wakefulness (Borbély, 1982) but also by the sensory overloading during the preceding waking period. In the latter case, prolonged sensory stimulation during waking seems differentially to affect not only neural mechanisms involved in the generation of specific brain activities during subsequent NREM sleep but also the structure of sleep as revealed by an increase in the total duration of SWS (Cantero et al., 2002).

At the neuronal level, EEG spectral power increases can be accounted for by either reductions in the phase shifts among the outputs of large cortical populations (Pfurtscheller and Lopes da Silva, 1999; Nunez et al., 2001) or by increments in the number of oscillations within a particular frequency range. Both neuronal behaviors have different physiological implications and should be precisely separated. We demonstrated here that those power increments restricted to $\alpha$ and spindles during SWS were particularly attributable to a generalized enhancement of the EEG amplitude, which may be attributed, among other factors, to elevations of the extracellular potassium in the neocortex (Louvel et al., 2001), changes in conductivity, or a high level of synchronization in the overstimulated cortical neurons. However, an increase in the wave incidence was also observed over auditory cortical areas for $\alpha$ and spindles, as well as over parietal regions regarding the sleep spindle band. These findings suggest that different cortical regions may be differently involved in the homeostatic brain responses during SWS after intensive activation of auditory pathways during wakefulness.

Increments of $\alpha$ power may result from increased activation of collaterals between neocortical columns containing intrinsic $\alpha$ oscillators (Lopes da Silva et al., 1973). Thalamocortical as well as long-range cortico-cortical coherence, both mechanisms being involved in the $\alpha$ activity generation (Lopes da Silva et al., 1980; Nunez et al., 2001), might facilitate the extent of this synchrony over wide cortical territories. On the other hand, spindle oscillations are generated by the combination of intrinsic properties and connectivity patterns of thalamic neurons (Contreras et al., 1997) synchronized over the neocortex through the action of both corticothalamic and cortico-cortical projections (Destexhe et al., 1999). The higher number of oscillations and larger wave amplitude selectively observed during SWS both at temporal and parietal cortices might be reflecting an increased flow of information through the thalamus to specific regions of the cortex to maintain a neural stabilization of cortical networks overused during the previous wakefulness. Accordingly, the massive calcium entry provoked by the excitation-inhibition input pattern on cortical pyramidal neurons simultaneously to the spindle oscillations has been suggested to underlie neural network reorganization after learning and/or sensory experience (Sejnowski and Destexhe, 2000). Likewise, the enhanced spectral power in the $\alpha$ range may also reflect a mechanism to favor synaptic efficacy. For instance, excitatory pulses of $10 \mathrm{~Hz}$ have been demonstrated to induce long-term potentiation in corticothalamic synapses (Castro-Alamancos and Calcagnotto, 1999), which might be explained by a facilitation effect on corticothalamic fibers from layer $\mathrm{V}$, in which $\alpha$ activity has been suggested to have its main neocortical generation sources (Lopes da Silva and Storm van Leeuwen, 1977). Thus, increments in EEG spectral power observed in the present study during SWS might be reflecting the 

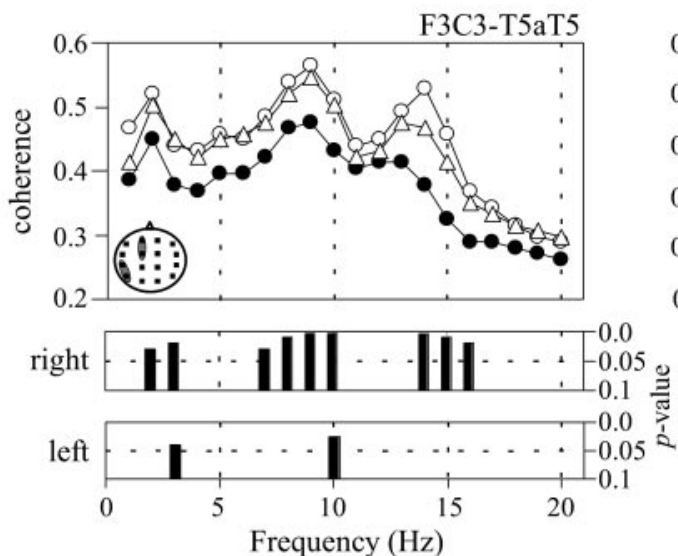
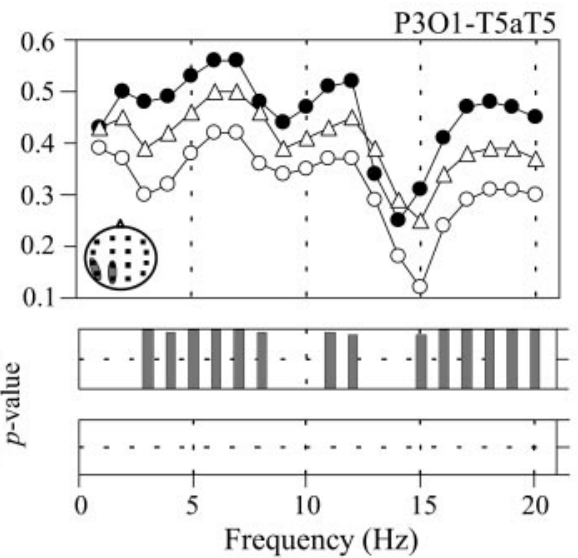

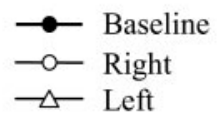

F3C3-T5aT5

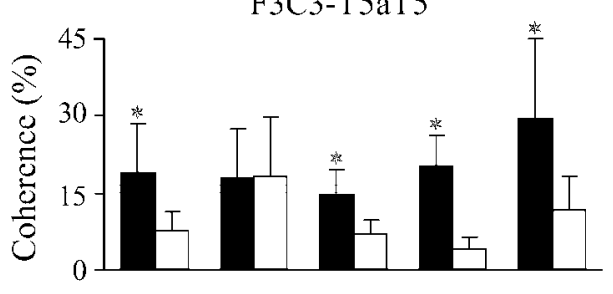

Delta Theta Alpha Spindle Beta
Experimental $>$ Baseline

\ Experimental $<$ Baseline
Figure 3. Mean EEG coherence values during SWS for those coherence pairs that showed significant differences in the overall rANOVA. right and left indicate the effects of the stimulation side on the EEG coherence during sleep. Bottom bar panels represent post hoc significance levels for comparisons between baseline and experimental (right or left) nights. Short-range coherence over the posterior left hemisphere (P3O1-T5aT5) decreased (gray bars), whereas long-range fronto-temporal coherence (F3C3-T5aT5) increased (black bars) after auditory stimulation over a wide spectral range compared with the baseline night. A schematic topographic representation of the coherence locations is depicted on the left bottom corner of each graphic.

\section{Right $\square$ Left}

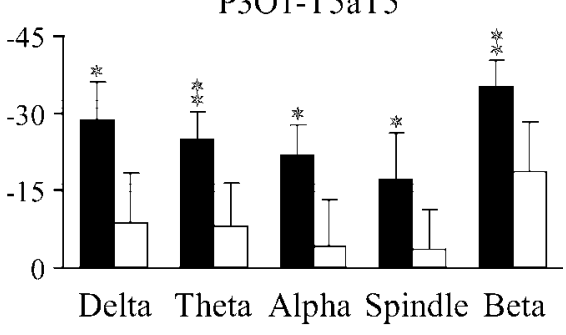

Delta Theta Alpha Spindle Beta

Figure 4. Mean and SE percentage of coherence change and in those coherence pairs (F3C3-T5aT5 and P3O1-T5aT5) in which the overall rANOVAs showed main effects of the night factor (baseline, right, and left stimulation) for each classic frequency band during SWS periods after either right or left acoustic overstimulation. Post hoc comparisons (baseline vs poststimulation night) for each coherence pair and frequency band are also reported $\left({ }^{*} p<0.05 ;{ }^{* *} p<0.005\right)$. Note that significant changes in cortical coherence were restricted to the night just after the first exposure to the auditory stimulation (right session). The direction of these changes in functional coupling between cortical areas was different in each case. Right auditory stimulation significantly weakened cortical coherence between left posterior cortex (P3O1) and left posterior temporal regions (T5aT5), whereas the coherence between left fronto-central cortex (F3C3) and left posterior temporal regions (T5aT5) were strengthened compared with the baseline night.

cortical reorganization after the intensive exposure to acoustic inputs.

The effects of the waking-auditory stimulation on cortical synchrony described above were widespread over the scalp, arguing against the local use-dependent theory of sleep function, which predicts an effect of auditory stimulation restricted to only those cortical regions involved in auditory processing. However, it is possible that the strong thalamocortical volleys reached other cortical populations, which were insufficiently stimulated during the preceding waking, manifest in the spatially diffuse $\alpha$ and spindle power increases not only over auditory areas but also over other regions of the cortex (central, parietal, and occipital). In this way, the brain might take advantage of the enhanced activity between previously overused circuits to differentiate them from those synapses that were not primed by previous stimulation. According to this view, Krueger et al. (1995) proposed that the main function of sleep is to maintain those structures insufficiently activated during wakefulness to avoid their atrophy. Thus, sleep would serve to preserve a constancy of a synaptic superstructure to brain organization and physiological regulation.

\section{Sensory experience-dependent plasticity as revealed by changes in cortical coherence}

Higher auditory structures that receive information of complex sounds also send projections to the frontal cortex (Rauschecker, 1998). Prolonged waking-stimulation of these structures might lead to local stimulus-dependent increases in metabolic demands and, consequently, to the release of adenosine and other SWSinducing substances within these populations (Benington and Heller, 1995). This release seems to cause hyperpolarization of cortical neurons, which, in turn, is directly related to synchronization of their bursting (Steriade et al., 2001). Prolonged wakingauditory stimulation might have facilitated the functional coupling within temporal and frontal neuronal populations. Accordingly, the increased synchronization of cell firing within each one of these populations may also enhance long-range synchronization between them to the detriment of interactions with other cortical regions as a consequence of the previous stimulation. This might be reflected by the enhancement of frontotemporal coherence during SWS periods and the coherence decrement between temporal and parieto-occipital regions in those nights after auditory stimulation. During SWS, the cortex exerts a powerful influence in grouping thalamic cells to fire synchronously through corticothalamic excitatory inputs (Steriade, 1997). Both $\delta$ and spindle synchronization between temporal and frontocentral corticothalamic firing should enhance their impact on thalamic nuclei. Consequently, thalamocortical output would also be stronger (Steriade et al., 1993), contributing to the strengthening of this long-distance feedback loop.

Unexpectedly, effects of the overstimulation on cortical coherence during SWS were restricted to the left hemisphere in the 
night after the first session of unilateral acoustic overstimulation. Competition between sensory inputs seems to be a necessary condition to produce generalized cortical changes after persistent sensory experience (Finnerty and Connors, 2000), which may account for the fact that overstimulation delivered on the right channel evoked changes selectively affecting the left hemisphere. This raises the question why stimulation presented to the left ear after 1 week produce no changes in cortical coherence over the right hemisphere. One possible explanation could be the lack of a novel component associated with the auditory stimulation patterns. This possibility is supported by recent evidence indicating that firing rate of CA1 pyramidal neurons are modified during sleep only when new sets of neurons are activated in a novel environment during the preceding waking (Hirase et al., 2001). Therefore, changes in the cortical coherence observed during SWS after prolonged exposure to stimulation might be a response to novel experience rather than to the cortical synaptic imbalance.

In summary, previous and present results confirm the fundamental role of exogenous factors, such as prolonged $(6 \mathrm{hr})$ sensory stimulation, on both the temporal organization of SWS (Cantero et al., 2002) and cortical activity pattern of the subsequent SWS periods. Enhanced spectral power within the $\alpha$ and spindle frequency range during SWS is interpreted as evidence of both cortical and corticothalamic homeostatic mechanisms in response to an excessive use of specific synapses associated to auditory processing during the preceding wakefulness. The fact that changes in cortical coherence were observed in a single night after the first stimulation session whereas changes in synchronization were equally obtained in the two nights after stimulation suggests that two different underlying brain mechanisms can be differentially activated after prolonged exposure to sensory experience. Our hypothesis is that electrophysiological changes revealed by modifications in the functional coupling between cortical regions reflect novel experience-dependent cortical reorganization rather than a single homeostatic response to the imbalance produced in the neuronal networks after sensory overstimulation, with SWS being the critical brain period for such synaptic reorganization.

\section{REFERENCES}

Benington JH, Heller HC (1995) Restoration of brain energy metabolism as the function of sleep. Prog Neurobiol 45:347-360.

Borbély AA (1982) A two process model of sleep regulation. Human Neurobiol 1:195-204.

Borbély AA, Baumann F, Brandeis D, Strauch I, Lehmann D (1981) Sleep-deprivation: effect on sleep stages and EEG power density in man. Electroencephalogr Clin Neurophysiol 51:483-493.

Cantero JL, Atienza M, Salas RM (2002) Effects of waking-auditory stimulation on human sleep architecture. Behav Brain Res 128:53-59.

Castro-Alamancos MA, Calcagnotto ME (1999) Presynaptic long-term potentiation in corticothalamic synapses. J Neurosci 19:9090-9097.

Contreras D, Destexhe A, Sejnowski TJ, Steriade M (1997) Spatiotemporal patterns of spindle oscillations in cortex and thalamus. J Neurosci 17:1179-1196.

Destexhe A, Contreras D, Steriade M (1999) Neocortical excitability controls the coherence of thalamic-generated oscillations through corticothalamic feedback. Neuroscience 92:427-443.

Essl M, Rappelsberger P (1998) EEG coherence and reference signals: experimental results and mathematical explanations. Med Biol Eng Comp 36:399-406.

Finnerty GT, Connors BW (2000) Sensory deprivation without competition yields modest alterations of short-term synaptic dynamics. Proc Natl Acad Sci USA 97:12864-12868.

García-García F, Beltrán-Parrazal L, Jiménez-Anguiano A, VegaGonzález A, Drucker-Colín R (1998) Manipulations during forced wakefulness have differential impact on sleep architecture, EEG power spectrum, and FOS induction Brain Res Bull 47:317-324.

Gasser T, Jennen-Steinmetz C, Sroka L, Verleger R, Möcks J (1988)
Development of the EEG of school-age children and adolescents. II. Topography. Electroencephalogr Clin Neurophysiol 69:100-109.

Hirase H, Leinekugel X, Czurko A, Csicsvari J, Buzsaki G (2001) Firing rates of hippocampal neurons are preserved during subsequent sleep episodes and modified by novel awake experience. Proc Natl Acad Sci USA 98:9386-9390.

Hoffmann RF, Moffitt AR, Shearer JC, Sussman PS, Wells RB (1979) Conceptual and methodological considerations toward the development of computer-controlled research on the electro-physiology of sleep. Waking Sleeping 3:1-16.

Huber R, Graf T, Cote KA, Wittmann L, Gallmann E, Matter D, Schuderer J, Kuster N, Borbély AA, Achermann P (2000) Exposure to pulsed high-frequency electromagnetic field during waking affects human sleep EEG. NeuroReport 11:3321-3325.

Jenkins GM, Watts DG (1968) Spectral analysis and its applications. San Francisco: Holden-Day.

Kattler H, Dijk D, Borbély AA (1994) Effect of unilateral somatosensory stimulation prior to sleep on the sleep EEG in humans. J Sleep Res 3:159-164.

Kavanau JL (1997) Memory, sleep and the evolution of mechanisms of synaptic efficacy. Neuroscience 79:7-44.

Krueger JM, Obál F, Kapás L, Fang J (1995) Brain organization and sleep function. Behav Brain Res 69:177-185.

Ktonas PY (1987) Period-amplitude EEG analysis. Sleep 10:505-507.

Lopes da Silva FH, Storm van Leeuwen W (1977) The cortical source of the alpha rhythm. Neurosci Lett 6:237-241.

Lopes da Silva FH, van Lierop THMT, Schrijer CF, Storm van Leeuwen W (1973) Organization of thalamic and cortical alpha rhythms: spectra and coherences. Electroencephalogr Clin Neurophysiol 35:627-639.

Lopes da Silva FH, Vos JE, Mooibroek J, van Rotterdam A (1980) Relative contributions of the intracortical and thalamo-cortical processes in the generation of alpha rhythms, revealed by partial coherence analysis. Electroencephalogr Clin Neurophysiol 50:449-456.

Louvel J, Papatheodoropopulos C, Siniscalchi A, Kurcewicz I, Pumain R, Devaux B, Turak B, Esposito V, Villemeure JG, Avoli M (2001) GABA-mediated synchronization in the human neocortex: elevations in extracellular potassium and presynaptic mechanisms. Neuroscience 105:803-813

Maquet P (2001) The role of sleep in learning and memory. Science 294:1048-1052.

Nunez PL, Srinivasan R, Westdorp AF, Wijesinghe RS, Tucker DM, Silberstein RB, Cadusch PJ (1997) EEG coherency. I. Statistics, reference electrode, volume conduction, Laplacians, cortical imaging, and interpretation at multiple scales Electroencephalogr Clin Neurophysiol 103:516-527.

Nunez PL, Silberstain RB, Shi Z, Carpenter MR, Srinivasan R, Tucker DM, Doran SM, Cadusch PJ, Wijesinghe RS (1999) EEG coherence. II. Experimental comparisons of multiple measures. Clin Neurophysiol 110:469-486.

Nunez PL, Wingeier BM, Silberstein RB (2001) Spatial-temporal structures of human alpha rhythms: theory, microcurrent sources, multiscale measurements, and global binding of local networks. Human Brain Mapp 13:125-164.

Pedemonte M, Peña JL, Torterolo P, Velluti RA (1997) Auditory deprivation modifies sleep in the guinea-pig. Neurosci Lett 223:1-4.

Pfurtscheller G, Lopes da Silva FH (1999) Event-related EEG/MEG synchronization and desynchronization: basic principles. Clin Neurophysiol 110:1842-1857.

Rauschecker JP (1998) Cortical processing of complex sounds. Curr Opin Neurobiol 8:516-521.

Rauschecker JP, Tian B, Hauser M (1995) Processing of complex sounds in the macaque nonprimary auditory cortex. Science 268:111-114.

Rechtschaffen A, Kales A (1968) A manual of standardized terminology, techniques and scoring system for sleep stages of human subjects. Los Angeles: UCLA Brain Information Service/Brain Res Institute.

Sejnowski TJ, Destexhe A (2000) Why do we sleep? Brain Res 886:208-223.

Steriade M (1997) Synchronized activities of coupled oscillators in the cerebral cortex and thalamus at different levels of vigilance. Cereb Cortex 7:583-604.

Steriade M, McCormick DA, Sejnowski TJ (1993) Thalamocortical oscillations in the sleeping and aroused brain. Science 262:679-685.

Steriade M, Timofeev I, Grenier F (2001) Natural waking and sleep states: a view from inside neocortical neurons. J Neurophysiol 85:1969-1985.

Tobler I, Franken P, Trachsel L, Borbély AA (1992) Models of sleep regulation in mammals. J Sleep Res 1:125-127.

Vyazovskiy V, Borbély AA, Tobler I (2000) Unilateral vibrissae stimulation during waking induces interhemispheric EEG asymmetry during subsequent sleep in the rat. J Sleep Res 9:367-371.

Webster DB, Popper AN, Fay RR (1992) The mammalian auditory pathway: neuroanatomy. New York: Springer. 